\title{
Investigating the Agrammatic Production of Canonical and Non-Canonical Sentences Cross-Linguistically
}

Mohammad Harun*

Comilla University, Bangladesh

Corresponding Author: Mohammad Harun, E-mail: harun.eng.du@gmail.com

\section{ARTICLE INFO}

\section{Article history}

Received: October 22, 2019

Accepted: December 11, 2019

Published: February 29, 2020

Volume: 11 Issue: 1

Advance access: February 2020

Conflicts of interest: None

Funding: None

\author{
Key words: \\ Bangla Agrammatism, \\ (Non-)Canonicity, \\ Passive Sentences, \\ Minimalist Program
}

\begin{abstract}
Research on agrammatism has revealed that the nature of linguistic impairment is systematic and interpretable. Non-canonical sentences are more impaired than those of canonical sentences. Previous studies on Japanese (Hiroshi et al. 2004; Chujo 1983; Tamaoka et al. 2003; Nakayama 1995) report that aphasic patients take longer Response Time (RT) and make more mistakes in producing non-canonical sentences compared to that of canonical sentences. The present research investigates the production impairments of canonical and non-canonical sentences cross-linguistically focusing on Bangla, Japanese, German and English aphasic patients. While Bangla, Japanese, German have relatively flexible word order, and hence allow freer phrasal movement, English exemplifies less freedom in word order patterns, and does not allow as much movement as the former three. We hypothesized that Bangla agrammatic patients would have more impairments in producing non-canonical sentences than those of canonical counterparts, while the production of canonical sentences is not completely devoid of impairments too. Primary data were collected from Bangla agrammatic patients, and secondary data from Japanese, German and English were exploited for cross-linguistic comparison. The findings show that Bangla agrammatic speakers have severe impairments in producing passive sentences, although the production of active ones are not completely devoid of impairments. The cross-linguistic comparison of the findings implies that the production of Bangla agrammatism tend to be similar to other agrammatic production and the production of non-canonical sentences are more difficult than those of canonical sentences cross-linguistically.
\end{abstract}

\section{INTRODUCTION}

Though Broca's area is usually considered to be the language production area, production of linguistic items is not exclusively confined to this region. Moreover, there is no $100 \%$ fine-grained interaction between area-division and language behavior. Till date, there have been many studies focusing on the functions of the different parts of the brain, and the severity of language impairments based on the damages in different parts of the brain studied. The studies include fMRI techniques, online, off-line language processing studies and others. Studies on non-canonical sentences i.e. object relative clauses, passive sentences, wh-interrogatives, etc. report that the sentence constructions that deviate from the basic word order are more difficult to process for agrammatic patients. As adult speakers over-learn the canonical sentences, they can produce the basic, canonical sentences comparatively easily. So far the previous research on agrammatism has reported that agrammatic speakers have specific types of language impairments.

Non-canonical sentences involve more computational load(s), and hence, agrammatic speakers face more challenges in producing these, yet the nature of the linguistic modalities involving more impairments in agrammatic production is not the same in all languages. To have a better understanding of the impairment modalities of canonical and non-canonical sentences cross-linguistically, the present study considers Japanese, German and Bangla from the list of relatively flexible word order languages, and English from the list of less flexible word order languages. Our purposes for involving languages from two poles of the continuum of word order flexibility are i) to report the production impairment(s) of canonical and non-canonical sentences of the respective languages, and ii) to make critical comparisons of the nature of impairments cross-linguistically.

The word order in Bangla canonical and non-canonical sentences may remain the same. If the aphasic patients face more difficulty in producing non-canonical sentences, it can be assumed that there are other extra-linguistic factors than only syntactic features for the patients finding the non-canonical sentences more difficult.

Moreover, the dearth of aphasia literature in languages other than English and Western European languages imbalances the universality of the theoretical underpinnings of the findings, "Some of the most widely spoken languages in the world, such as Arabic, Hindi, Bengali, Russian, as well 
as Portuguese, accounted for less than $0.5 \%$ of the aphasia literature. This imbalance has not improved over the examined time period; in fact, the percentage of papers on English seems to be increasing" (Beveridge, 2011). This is why we undertook the present investigation. Keeping the above issues in mind, the present study seeks to address the following questions:

i. Are there any production differences between canonical and non-canonical sentences in Bangla agrammatism?

ii. Why do aphasics face more difficulties in producing non-canonical sentences?

iii. How do the additional computational loads i.e. identification of traces of the moved constituents, Topic Phrase etc. of the non-canonical sentences account for the added difficulties in agrammatism?

iv. Do deviations from both base word order and case make sentences more difficult to produce?

\section{Hypotheses}

H0 - There will be no difference in agrammatic production of canonical and non-canonical sentences in Bangla agrammatism.

H1 - Bangla Agrammatic production of non-canonical (passive) sentences will be more impaired than those of canonical (active) sentences.

\section{LITERATURE REVIEW}

Studies on agrammatism have attracted researchers from a wide range of subject expertise i.e. linguistics, neurolinguistics, computational linguistics, psycholinguistics, communication disorders etc. Till date, interesting observations and hypotheses centering the interaction between the different parts of the human brain and different linguistic behaviors are established. More interestingly, accurate identifications of the specific parts of the brain and the specific linguistic features are enriching the fields of theoretical linguistics day by day and the subject-matters of its several applied branches i.e. neurolinguistics, computational linguistics, psycholinguistics, communication disorders etc. are expanding rapidly. Against this backdrop, we aim to analyze cross-linguistically the production impairment of canonical (active) and non-canonical (passive) sentences of aphasics.

\section{Theorization on Agrammatic Production}

The results of many studies on agrammatic production (Avrutin, 2001), Agnew et al. (2014), Lee and Thompson (2005), Arabatzi \& Edwards, (2002), Kok \& kolk (2007), Fyndanis et al. (2012), Rispens et al. (2001), Kemmerer (2012) and Engel et al. (2017) show that agrammatic production is more or less impaired across different modalities. Though it is yet not resolved whether impairment of agrammatic production is due to representational deficits or processing deficits, investigations are being conducted to determine the nature of agrammatic production. Many studies subscribe to the theory of processing deficits, rather than the representational account for agrammatism.
Cho-Reyes \& Thompson (2012) report agrammatic groups producing overall more incorrect responses with their studies on 35 individuals with agrammatic aphasia, and 24 with anomic aphasia using the Western Agrammatic Battery (WAB). The study reports that the more the number of arguments was, the less sentence accuracy was found, while prepositional impairments were the severest (Cho-Reyes \& Thompson, 2012). Agrammatic production is characterized by absence of grammatical markers, and syntactic structure, morphological deficits \& constructional impairments. Schwartz et al. (1987) suggest that patients with agrammatism have difficulty in mapping relations between the abstract functional level and the surface syntax at the positional level. Below, we discuss some prominent hypotheses that developed from research on agrammatism.

\section{Tree pruning hypothesis (TPH)}

Tree Pruning Hypothesis (TPH), as Friedmann and Grodzinsky (1997) phrases, simply refers to the fact that some parts of the syntactic tree become inaccessible to the agrammatics i.e. some parts are 'pruned'/impaired resulting in omission of certain grammatical items. The impairment may be caused at higher or lower nodes of the tree depending on the severity of impairment. It also predicts that usually the higher nodes are more impaired than the lower nodes, but not vice-versa, and the impairment is selective. The predictions of TPH are as follows:

1. i. The highest node, $\mathrm{CP}$, is always impaired to some degree

ii. Material at higher nodes are more impaired than material at lower nodes, but not vice versa

iii. Tense inflection being higher is more difficult to produce than agreement inflection

Friedmann and Grodzinsky (1997) and Friedmann (2002) report that their Hebrew and Arabic speaking patients produced more tense errors than agreement errors (Cited in Duman, 2009, p. 7). Not all studies on TPH report similar results such as, the predictions of TPH are not supported by the experiments that Lee and Thompson (2005) conducted on production of (in Experiment 1) complementizers (if, whether, and that) and (in Experiment 2) of verb inflections (3rd person singular $-s$, present plural bare stem $-\theta$ and past tense -ed). They report that $\mathrm{CP}$ remains intact while the TP and AgrP are impaired. Their experiments with FG and LC report that FG and LC produced 19 and 20 correct responses respectively out of 20 complementizer task items. They report of tense being more impaired than any other nodes (contra the predictions that $\mathrm{CP}$ would be mostly impaired) while AgrP is reported to be the least impaired node (both patients performed better in agreement than tense). As there is no unanimity with regard to the position of AgrP in IP, the results are yet inconclusive for the syntactic-tree-based account. Pollock posits AgrP below tense phrase in the split IP, while both Ouhalla (1993), and Chomsky (1993) place subject agreement above, and object agreement below tense phrase in GB/Principles and Parameters Framework. Chomsky (1993) also differentiates between subject agreement and object agreement and puts the tense phrase in 
between these two, while Ouhalla (1993) opines for a common agreement phrase situated above tense phrase.

\section{Derived order problem hypothesis}

Another interesting theory i.e. Derived Order Problem Hypothesis (DOP-H) holds that all languages have a base word order such as English has SVO, Bangla, Dutch and German have SOV as base word order. When agrammatic patients encounter sentences with the word order in derived position i.e. moved from the original position, they find the sentences more difficult to produce than the sentences with the base word order. Bastiaanse et al. (2002a) report, "Dutch patients have more problems with (finite) verb inflection and/or word order in main clauses than in embedded clauses (Cited in Duman, 2009, p. 11). Duman (2009) also points, "Note that the DOP-H is restricted to word order."

\section{Extra-linguistic factors for processing limitations}

Kok et al. (2007) investigate whether the representational models or the processing limitations (e.g. limited working memory capacity) are responsible for impairment of agrammatic language production. Their findings match with that of the account of the processing limitations hypothesis as is proposed by Avrutin (2001). The processing deficits hypothesis claim that agrammatic impairment is the result of the subjects' processing limitations of complex, non-canonical sentences. The processing hypothesis assumes that tense inflection is comparatively difficult to produce for its being computationally complex that requires "integration of information at grammatical and conceptual level" (Avrutin 2001). This becomes problematic for agrammatic patients. Integration of grammatical and discourse level representations makes it difficult for the patients to process information. Kok et al. (2007) comment that their findings imply that the difficulties in producing verb/tense inflection cannot be explained by a representational account. Hartsuiker et al. (1999) record agrammatics' as well as normal speakers' approach to complete the following sentences with singular verb inflections:

2. a. The baby on the blankets

b. The label on the bottles

As sentence 2.a. contains an NP that is singular both at the grammatical and conceptual level, normal speakers took both accounts to complete agreement inflection but made more agreement errors in sentence 2.b. which is grammatically singular but conceptually plural. Agrammatic speakers do not account for conceptual meaning, but only grammatical account to avoid extra computational load for integrating conceptual information. So, the findings do not support the representational account which is only grammatical, but processing account that integrates both grammatical and discourse information. Kok et al. (2007) show patients making more errors (both in tense and agreement inflections) when (computational) performance load are double in Order and Inflection Test than single (computational) performance load in Inflection Test.

The group patterns of the results also do not support Tree Pruning Hypothesis (Friedmann \& Grodzinsky, 1997) nor Tense Underspecification Hypothesis (Wenzlaff \& Clahsen, 2004). The effects of computational load cannot be accounted for by the representational hypotheses such as $\mathrm{TPH}, \mathrm{TUH}$, rather are captured by processing limitations as the agrammatic performances in dual/single task complexity show. The tense node being higher in the syntactic tree than the agreement node, impairment in agreement leads to tense inflection at chance level performance. Because there are both pure tense inflection errors as well as pure agreement inflection errors that depend on the complexity of the task at hand.

Not all the studies yield consistent data as many hypotheses and results are found to be refuted in later studies. Shapiro et al. (1987) report of normal speakers' taking longer time for processing verbs that have more possible argument structures (e.g. alternating datives such as 'send' takes more resources than a transitive verb such as 'fix'). In this regard, Broca's aphasics show their sensitivity to a verb's representational complexity, whereas, Wernicke's aphasics failed to do the same.

\section{Feature interpretability \& minimalist program in agrammatism}

Fyndanis et al. (2012) report of selective impairment in verb inflection based on feature interpretability and align the findings with the Minimalist Program (Chomsky, 1995). Elements that have discourse representation alongside the grammatical ones are interpretable, contra, elements that have only grammatical representation, and have no discourse representation, are uninterpretable features. Nanousi et al. (2006) and Varlokosta et al. (2006) report of Aspect being more impaired than Agreement in Greek agrammatism, though Aspect is lower than Tense and Agreement in the syntactic tree in Greek. Fyndanis et al. (2012) propose Interpretable Features' Impairment Hypothesis (IFIH) claiming that interpretable features, such as Tense and Aspect are more impaired than Agreement which is not an independent functional category but an operation by which certain uninterpretable features of Tense are checked against certain interpretable features. They propose that the syntactic process that assigns phonological values to interpretable features is impaired in their agrammatic speakers. Bastiaanse (2008), Duman and Bastiaanse (2009) and Bastiaanse et al. (2011) report that the reference to the past is more impaired than the reference to present or future. But Fyndanis et al. (2012) find no difference in difficulty regarding reference to past, future or present. Their results also do not support TPH, rather subscribe to the proposal of Nanousi et al. (2006) and Varlokosta et al. (2006) that is interpretable features, such as Tense and Aspect being more impaired than uninterpretable features, such as Agreement. Their results are also at par with the proposal of Faroqi-Shah and Thompson (2004 $\& 2007)$ that claim agrammatic speakers have more impairment in encoding $\mathrm{T}$ related diacritical features and retrieving corresponding verb forms. The findings are also in line with the spirit of TUH and TAUH as Tense is found more impaired than Agreement. 


\section{Agrammatic production of non-canonical sentences}

Whether production impairments are results of representational deficits or processing deficits are yet an ongoing debate. Burchert et al. (2008) study the production of non-canonical sentences in German. They experiment to know whether Tree Pruning Hypothesis (TPH) or Derived Order Problem (DOP-H) hypothesis account for agrammatic production. It also holds that when a node of the tree is impaired, the upper nodes from that particular node are also necessarily impaired. On the other hand, DOP-H looks upon the impairment as "limitations in the application of movement rules" (Burchert et al. 2008). When there occurs any movement in the syntactic tree, aphasic patients find the processing of the moved object difficult, and the greater is the distance between the moved object (filler) and the place from where the NP has been moved (gap or trace), the more difficult is the production for agrammatic speakers. For this reason, certain sentences that involve movement (i.e. non-canonical sentences) are more difficult to produce by agrammatic patients than the sentences that do not involve movement (i.e. canonical sentences). In this regard, passive sentences and object-relative clauses, interrogatives, etc. best exemplify non-canonicity in any particular languages, as such constructions involve derived word order.

Burchert et al. (2008) find that Broca's agrammatic patients had greater difficulty and more errors with the production of scrambled (non-canonical) sentences. Additionally, object-relatives, wh-interrogatives, passives are reported to be more difficult to produce. Their findings support the DOP-H rather than the TPH. Their discussion does not shed light on the distinction between Movement and Scrambling and considers both phenomena in the same spirit i.e. non-canonical. The comparison between canonical and non-canonical sentence production shows that group performance was more difficult for non-canonical sentences than canonical sentences. Additionally, the main source of errors was descrambling sentences (error type 1) when scrambling was required. Their results of non-canonical object-adverb order are in line with Bastiaanse et al.'s (2003) study with Dutch-speaking agrammatic patients. Their results support the DOP-H hypothesis as they find problems with sentences involving movement, not with sentences with $\mathrm{CP}$ or higher node impairment.

Makuuchi et al. (2012) focus on the processing of non-canonical sentences in the left hemisphere in Broca's area in their fMRI study. They report that "the longer the filler-gap distance, the greater the difficulty in reaccessing the filler from the lexicon." The distance between the filler and the gap increases the processing demands from both syntactic and semantic points of views as both syntactic-semantic features of the filler need to be maintained until the gap is met. In the case of Scrambling in German, a linear increase of activation is well-documented and results that are reported maintain consistency (Makuuchi et al. 2012; Friederichi et al. 2006). Additionally, Makuuchi et al.'s (2012) study find the "nonlinear increase in the Movement construction in German" as a new phenomenon. Their findings also show that "neural network consisting of the PO, IFS,
mMTG, and IPS" is "involved in the processing of filler-gap relations in non-canonical sentences constructed either by Movement or Scrambling operations" (Makuuchi et al. 2012). While the neural basis for the filler-gap is same for both conditions, there is a slight quantitative difference in the linear effect of distance (Makuuchi et al. 2012).

The above study also finds that the left inferior frontal gyrus (IFG), PO, PTr and IFS and the mMTG and IPS are activated due to the effect of distance between the filler and the gap. "The IFG and the mMTG in the left hemisphere are often co-activated in sentence processing and comprise a core language network to compute linguistic information" (Roder et al. 2002; Bornkessel et al. 2005; Hoen et al. 2006; Vigneau et al. 2006; Newman et al. 2010; Price 2010; Friederici et al. 2011). "Of these 2 regions, the IFG has more reliably been activated by filler-gap distance" Friederici et al. 2006; Santi and Grodzinsky 2010).

Faroqi-Shah \& Thompson (2004) find impairments with passive sentence production by English speaking agrammatic subjects. They further report that "the difficulties were not overcome when lexical cues (the relevant nouns and uninflected verb) were provided" (Faroqi-Shah and Thompson, 2004). But providing the auxiliary and past tense morphemes along with the stem verbs improved the production of passive construction significantly. This suggests that retrieving and processing the relevant grammatical forms were the causes for difficulty in producing passive sentences. Faroqi-Shah \& Thompson (2004) also report of agrammatic speakers' resorting to "a default tendency to produce active sentences because they are the most frequent form in English" (also in many other natural languages.) Considering all these, we also predicted that Bangla speaking aphasic subjects would hardly produce passive sentences, as passives in Bangla are not used that much in everyday communication.

Levelt (1999) considers that the syntactic words (lemmas) are activated by the lexical message and this lexical-syntactic information "builds up the appropriate sentence pattern, the surface structure" (p.88). The syntactic frames of different verbs (i.e. of active, passive, intransitive) are lexically represented as different lemmas (Levelt, 19999) and determine thematic roles accordingly. So, the syntactic frame of the passive lemma is: Verb ${ }_{\text {passive }}$ subject ${ }_{\text {Theme }} \mathrm{V}_{\text {Object }}$ optional ${ }_{\text {Agent }}$, and the syntactic frame of active lemma is: Verb ${ }_{\text {active }}$ subject $_{\text {agent }}$ $\mathrm{V}_{\text {objecttheme }}$. Similarly, the verb morphology of passive lemma is auxiliary $\mathrm{V}_{\text {Participle }}$. Kinno et al. (2017) report of comprehension difficulty by agrammatic patients with non-canonical sentence structures. They find agrammatic patients performing worse in non-canonical constructions, whereas, the performance accuracy with canonical sentences were not significantly different from normal age-matched subjects.

Being inspired by the results of previous studies on the production impairment of canonical and non-canonical sentences, we focused only on the production discrepancy between canonical, (active) sentences and non-canonical (passive) sentences in Bangla in the present study.

\section{Previous Studies on Bangla Agrammatism}

So far we know that there is dearth of studies on the production differences between canonical and non-canonical 
structures in Bangla. Though Tamanna (2017) has conducted research on the comprehension of derived word order i.e. non-canonical structures, she did not focus on the production impairment of non-canonical sentences. Imtiaz (2013) has conducted research on Bangla agrammatism. But neither studies focus on the production impairment of canonical and non-canonical sentences.

Tamanna's (2017) study focuses on the scrambled sentences in Bangla agrammatism. The results confirm the existence of agrammatism, and suggest that Bangla agrammatic speakers' production of sentences of derived word order is impaired more than that of basic word order sentences. But she did not consider the factors responsible for having more impairment with non-canonical sentences, especially with passives and object relative clauses. We could not reach any decisive conclusion(s) concerning the reasons for committing more mistakes/errors with the non-canonical structures. Imtiaz (2013) focuses on the comprehension deficits of Bengali speaking Broca's aphasics. He tests the trace deletion hypothesis (TDH) to verify the actual state of syntactic agrammatism. The results of this research reveal that the comprehension of canonical active sentences of Bengali aphasics is intact compared to the comprehension of other sentence structures. But we could not know whether the impairment is caused due to structural deficits or processing limitations. Keeping this in mind, the present study aims at investigating the agrammatic production of canonical and non-canonical structures of Bangla.

\section{METHOD}

\section{Linguistic Stimuli and Other Materials}

A questionnaire with a set of 30 picture-stimuli-sentence-production prompts was presented to both agrammatic speakers and age-matched control group. We divided the linguistic stimuli into two categories: a) Uncued picture-sentence production prompts, b) Cued picture-sentence production prompts. For uncued active-passive sentence production, picture-sentence production tasks were used. For this task, no linguistic stimuli i.e. the subject(s)/Agent(s), objects and verb(s) were provided. We provided the subjects only the stimulus-pictures. The cued picture-sentence production task was used with the Bangla subject(s)/agent(s), objects(s) and verb(s) randomly provided in uninflected forms. Subjects were required to produce sentences using these correctly. Subject(s), objects and verb(s) were provided to reduce the production pressure of any other lexical contents but only the movement related difficulties.

\section{Subjects}

Ten monolingual Bangla speaking agrammatic aphasic patients and ten monolingual control subjects were interviewed for the present study. More patients were approached, but many were dropped from the study as they could neither complete the tasks nor respond to the stimuli either verbally or in written. One patient had damage to the right hemisphere of the brain. All the patients were diagnosed with CVD. All the patients were right-handers. While the etiology of the stroke of seven subjects was identified HTM, the other three patients had ITM. Ten neurologically intact controls were chosen for the present experiment to counterbalance the findings.

\section{Procedure}

For procedural convenience, we divided the data collection, analysis etc. into two segments i.e. Experiment 1 (Production of uncued active-passive sentences), Experiment 2 (Production of cued active-passive sentences). Two sets of data were collected in two sessions- i) uncued picture-sentence production data of active and passive sentences, ii) cued picture-sentence production data of active and passive sentences, from both control group and experimental agrammatic aphasic speakers. Due to both mental and physical vulnerability of the patients and for ensuring reliability in the data collection processes, we arranged for two different sessions for the interviews with each patient. Task completion stimuli were presented randomly to both normal group and aphasic patients to control any effects arising from presenting the stimulus. The subjects were presented the linguistic stimuli with PowerPointPresentation software using a 15-inch laptop.

\section{FINDINGS}

We categorized the findings according to the types of impairment(s) across the syntactic (structural) criteria i.e. canonical and non-canonical sentences. We interviewed ten stroke-induced agrammatic patients with two sets of questionnaire in two sessions. We elicited a total of 560 responses from ten monolingual Bangla speaking agrammatic aphasic patients and also the same number of responses from ten controls.

\section{Error Analyses}

Based on our analyses, we identified seven types of errors e.g. i) Choosing other lexical verbs, ii) Grammatical morpheme errors, iii) Single word production, iv) Producing Non-sentences, v) Role-reversals, vi) Unrelated sentences, and vii) Producing active sentences in lieu of passives. Error type vii applies only for passives i.e. when subjects produced active sentences in lieu of passives, we considered those as errors since the instructions were given explicitly to produce passive sentences.

This error identification revealed a significant fact that not all types of errors occurred with similar frequency. We observed that agrammatic subjects had more difficulty in producing passive sentences. And subsequently, in our analyses, the most frequently occurring error type was producing active sentences where the production of passives was required. This single error pattern was $53 \%$ of the total errors. This pattern was observed in both Uncued Picture-Sentence Production and Cued Picture-Sentence Production tasks.

\section{Findings and Analyses of Results of Errors in Different Linguistic Items}

i) Production Errors of Uncued Canonical (Active) and Non-Canonical (Passive) Sentences: 

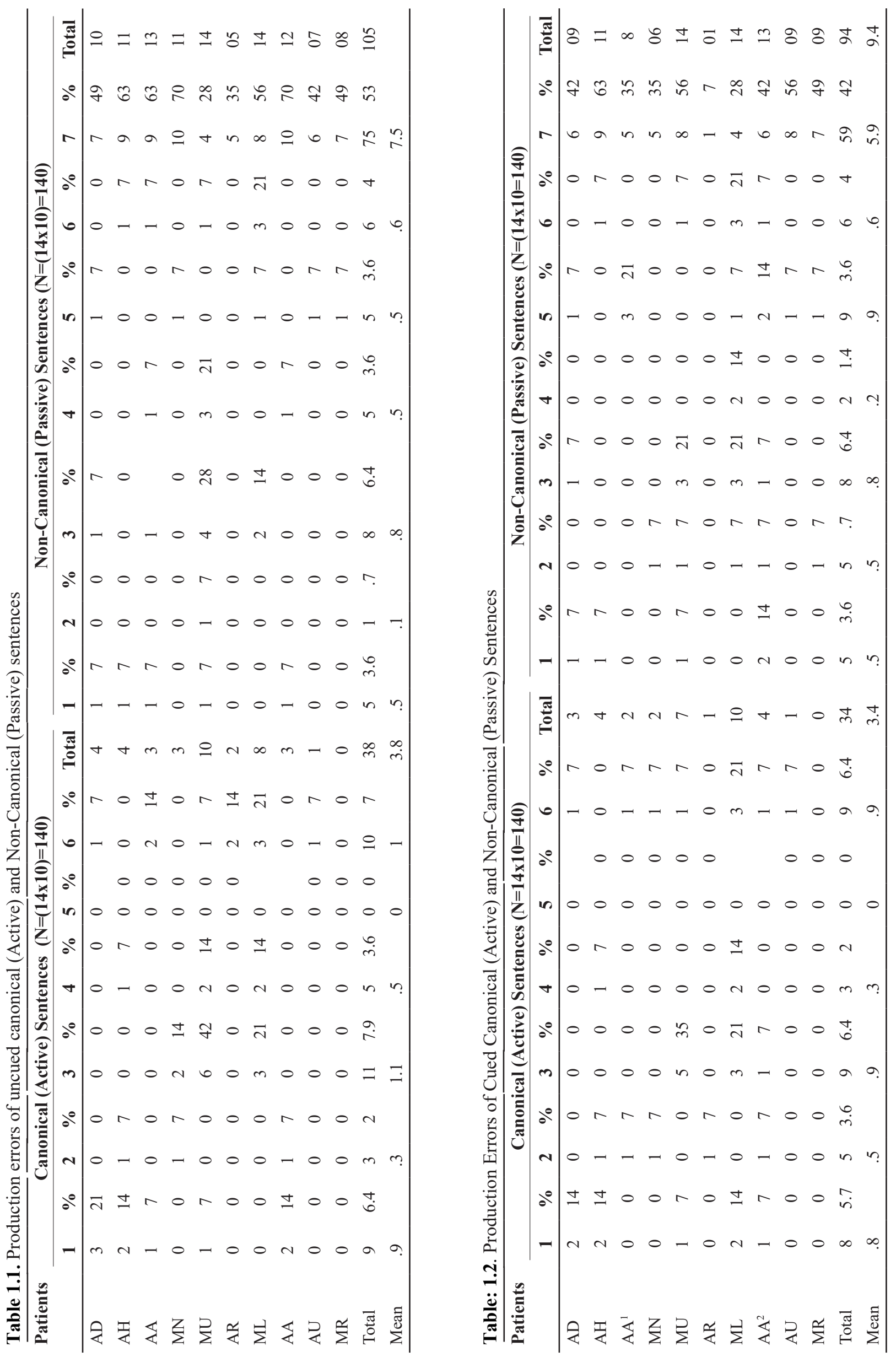
The findings of Experiment 1 that is Unceud Picture-Sentence Production show that agrammatic aphasics produced a total of $143(59.6 \%)$ errors both in the active (canonical) and passive (non-canonical) sentences. Of the total number of errors, $105(75 \%)$ were made on passive sentence production. And of these, one type of error i.e. producing active sentences instead of passives was the most difficult item to the patients ( 75 errors which are $52.9 \%$ of all passives). The second highest number ( 6 errors) in any single error type, which is much less than the highest number though, was 'unrelated sentences'. 'Role-reversal' and 'choosing other lexical words' were the third frequent number of errors (i.e. 5 errors for each category). On the passive sentence production task, MU and ML produced the highest number of errors i.e. 14 , followed by $\mathrm{AA}^{1}$, who made just one error less than MU and ML e.g.13 errors. We observed flooring effect in the performance of these three subjects. Though we noticed that 'producing active sentences instead of passives' was the most frequent error type, the three patients, who showed flooring effect, did not produce that many active sentences compared to others. On the other hand, AR had the least errors on both active sentence (02) and passive sentence production (05) tasks. All 5 errors that AR made were one type of error i.e. 'producing active sentences instead of passives'. AU made one error in active sentence production task, while there were 7 ( 1 role-reversal +6 producing active sentences instead of passives) errors on the passive sentence production task. On the active sentence production task, ten subjects produced a total of $38(27 \%)$ errors. The results clearly show that the production of passives (non-canonical) is more impaired than that of active sentences in Bangla agrammatism. Now we turn to the production errors of Cued Picture-Sentence Production experiment to see whether there is improvement in production when uninflected NPs and VPs are provided as cues.

ii) Production Errors of Cued Canonical (Active) and Non-Canonical (Passive) Sentences:

1 - Choosing other lexical verbs/words

2- Grammatical morpheme errors

3 - Single word production

4 - Producing Non-sentences

5- Role-reversals

6- Unrelated sentences

$7^{1}$ - Producing active sentences in lieu of passives

The results of Cued Picture-Sentence Production task show that agrammatic subjects produced fewer errors, but the number of errors reduced very slightly when uninflected NPs and VPs were provided as cues. Ten subjects made a total of $34(24.3 \%)$ errors on active sentence production, and $94(67 \%)$ errors on passive sentence production out of total 140 items for each category. The frequency of errors reduced from 38 to 34 (only $2.9 \%$ ) on the active sentence, and from 105 to $94(7.9 \%)$ on the passive sentence production when the cues were provided. The results are at per with previous studies (e.g. Faruqi-Shah et al. 2003) that aphasics did not significantly improve production when cues were provided. On both uncued and cued production conditions, the highest errors occurred in passive sentence production. On the active sentences, unrelated sentences were the most impaired linguistic items (9 errors), whereas, on passives, the highest (59, or $42 \%$ ) errors occurred when subjects produced active sentences instead of passives. This tendency proves that active sentences are the canonical structures and over-learnt linguistic items in Bangla.

vii) Summary results of both experiment and control groups' Production in tabular form:

The summary results show that the agrammatic subjects produced a total of 271 errors, and the controls made 83 errors out of 560 responses in all three experiments. Of the six subcategories of linguistic items i.e. uncued active, uncued passive, cued active, cued passive, the aphasics produced 105 errors in uncued passive sentences which is the highest number of errors. Production of passives proved to be most difficult, even when uninflected cues were provided (the experiment subjects made 94 errors in this regard). Occurrences of errors did not lessen much when cues were provided in the cued active sentence items. The aphasics lessened errors from 38 errors in the uncued active sentence to 34 errors in the cued active sentence items, on the other hand, the controls produced significantly fewer errors in the cued passive sentences ( 28 errors) than in the uncued passive sentences (40 errors)

\section{DISCUSSION}

The findings with regard to the canonical structures show that these are comparatively easy to produce. Table 1.3 shows that $27 \%$ errors are detected with the group responses for uncued canonical active sentences, while the percentage dropped to 24 with cued canonical active sentences. At the individual level, AU made total 10 errors on active sentences, while AU as well as ML could produce not a single correct response on the subject relative sentences. Also, his impairment of non-canonical sentences is significant (14 on the passives). In the non-canonical sentence production task, the group performance showed significant complexity (49.7\%). Overall production impairments for non-canoni-

Table 1.3. Summary results of both experiment and control groups' production of all linguistic items

\begin{tabular}{|c|c|c|c|c|c|c|c|c|c|}
\hline Patients & $\begin{array}{l}\text { Linguistic } \\
\text { Item }\end{array}$ & $\begin{array}{c}\text { Total } \\
\text { Responses }\end{array}$ & Errors & $\%$ & Controls & Linguistic Item & $\begin{array}{c}\text { Total } \\
\text { Responses }\end{array}$ & Errors & $\%$ \\
\hline & Uncued Active & 140 & 38 & 27 & & Uncued Active & 140 & 8 & 5.7 \\
\hline & Uncued Passive & 140 & 105 & 75 & & Uncued Passive & 140 & 40 & 28.6 \\
\hline & Cued Active & 140 & 34 & 24 & & Cued Active & 140 & 7 & 5 \\
\hline & Cued Passive & 140 & 94 & 67 & & Cued Passive & 140 & 28 & 20 \\
\hline & & Total: 560 & Total: 271 & 48.4 & & & Total: 560 & Total: 83 & 14.8 \\
\hline
\end{tabular}


cal passive sentences were in line with previous findings in Japanese, German and English that report of passives being more difficult to produce than their active counterpart canonical sentences.

When the subjects were provided with uninflected cues for the active-passive sentence production task, sentence accuracy improved but slightly. This was significant (error reduced from $27 \%$ to $24 \%$ ) for the active sentences. But for the production of passive sentences, the accuracy improved but very slightly (errors reduced from $75 \%$ to $67 \%$ ). Subjects tended to produce more active sentences in lieu of the passives. Moreover, most of the sentences from the passive sentence production group that were considered errors were actually attempted to produce active sentences. Within these active sentences, some sentences, even though, they were correct (grammatically), were considered errors as these were produced where the production of passive sentences was required. The present findings tend to comply with some previous studies (Faroqi-Shah \& Thompson, 2004; Burchert et al., 2008; Bastiaanse et al., 2003; Makuuchi et al., 2012; Levelt, 1999).

Burchert et al., (2008) found more impairment with non-canonical sentences with German speaking aphasics. Noticeable in that study was that agrammatic speakers find the production of reversible passives more difficult than non-reversible passives. Bastiaanse et al. (2003) also report of impairment with sentences involving movement. More importantly, Makuuchi et al.'s (2012) famous fMRI study reported, "...the longer the filler-gap distance, the greater the difficulty in reaccessing the filler from the lexicon." This happens due to the additional demands that need to be maintained till the gap is met. Levelt's (1999) account of different verbs having different lemmas is also relevant here. As the lemmas of active and passive verbs are different, the thematic $(\theta$-Role) role assignment and verb morphology are also different - the verb morphology of passive lemma always involves an additional auxiliary verb. This involves extra difficulty in the computational processes of the passive sentences.

In line with Faroqi-Shah \& Thompson's (2004) study, Bangla speaking aphasic patients showed noticeable production impairment of both reversible and non-reversible passive sentences. We consider both Faroqi-Shah \& Thompson (2004) and the present study as representative, because we modelled the data collection processes following the former study. In the first phase, we elicited responses without giving any lexical cues, but only the picture stimuli. In the second phase, we provided the patients with lexical cues to see if that had any effect(s) on the production. Therefore, both studies make the claim stronger that the active sentences are canonical sentences, whereas, the passive and other derived sentences are non-canonical structures. There was a significant difference between the production of reversible and non-reversible sentences. Reversible passive sentences were less accurately produced than the non-reversible passive sentences.

Interestingly, not any subject produced either active or passive sentences with past tense morphology. Producing past tense morphology was not required for the present study too. So, we refrain from further comment on the impairment of past tense morphology in Bangla aphasic agrammatism. The present study differs from Faroqi-Shah \& Thompson's (2004) study in the sense that, unlike Faroqi-Shah \& Thompson's (2004) study, the present study provided only uninflected cues for both active and passive sentences, whereas, FaroqiShah \& Thompson's (2004) study provided passive verb cues i.e. "was pushed" (Faroqi-Shah \& Thompson, 2004). It is generally assumed, and also studies report, that agrammatic speakers find it difficult to produce past tense morphology as reference to past time appears to be difficult for them. The present study cannot talk about the impairment of past tense by agrammatic speakers as we did not design our study to investigate Bangla past tense morphology impairment. But as subjects did not produce any past tense sentences even by chance, we can only insinuate that sentences in present tense might be the most frequent structures for agrammatic speakers as well as reference to the present time in Bangla.

\section{Impairment with Bangla Passives}

From the present study, we identified some significant facts. Our analyses showed that aphasic patients produced the highest number of errors with uncued passives ( 75 errors). The number of total errors lessened, but slightly (from 105 in the uncued condition to 94 in the cued condition) when uninflected NPs and VPs were provided in the second experiment (Cued Picture-Sentence Production). In all forms of sentence production, subjects found production of passives most difficult. The subjects mostly produced active sentences when production of passives was required. This increased the number of total errors too, as we considered any sentences produced as active (either grammatical or ungrammatical) when passives were required as incorrect. Aphasic subjects produced total 271 (48.4\%) errors out of total 560 responses. At the group level, the control group also did not reach any ceiling effects as they ended up producing total 83 errors out of 560 responses. Agrammatic subjects produced the highest number of errors (105) in uncued passives. The second highest number of errors occurred in cued passives (94 errors). The error patterns establish the fact that the production of passives was most difficult for the aphasic patients.

The present findings are in line with many previous studies. Faroqi-Shah \& Thompson's (2004) study with English speaking Broca's and Wernicke's patients report similar results. Both Broca's and Wernicke's patients had difficulty with passive sentence production, and the impairment was not less even when nouns and uninflected verb forms were provided. The present study also modeled similar production elicitation tasks i.e. uncued-picture-stimuli-production task and cued-picture-stimuli-production task for investigating the active-passive sentence production dichotomy in Bangla.

The findings are also compatible with Mehri et al.'s (2016) findings of Persian agrammatism. The study reports that Persian speaking non-fluent aphasic individuals found the production of non-canonical sentences more difficult than canonical sentences.

Passive sentences are both grammatically complex and semantically non-canonical. From the syntactic point of view, passive sentences usually involve the movement of 
noun phrases plus insertion of the additional passive-marking particle(s), such as by in English, dwara/diye/kortrik in Bangla, von mir in German etc. And from the semantic point of view, the object NP becomes prominent and moves to the subject position for passivization in English and German. But in case of Bangla, the canonical SOV structure is maintained even in passives, and there occurs no movement or displacement of the NPs i.e. Agent NP does not move to object position and object NP also does not move to subject position. Yet passives in Bangla are considered non-canonical due to the semantic complexity of Bangla passives.

We can claim that though passives in Bangla do not involve NP-Movement, passives serve complex functions at the conceptual level. Moreover, as the pragmatic domains of passives are also restricted in Bangla, subjects found the production of passives more difficult than active sentences. In this regard, we cannot say that impairments of passives are caused due to the DOP-H that holds that any sentence structures that are derived from the basic structures appear to be more difficult. The impairments should be for any other causes too along with syntactic non-canonicity. Additionally, passives in Bangla are pragmatically very constrained i.e. are very infrequent in everyday spoken language. This might be one of the causes for the patients' finding the production of passives more difficult. Secondly, from the syntactic point of view, Bangla passive sentences, like the passives in other languages, contain more syntactic elements, and semantically complex thematic relations.

Regarding the processing of passives, there are in vogue two accounts i.e. Movement-based accounts and Head-Driven Phrase Structure Grammar. Movement-based accounts advocate of triggering syntactic reanalysis of passives. Mack et al. (2013) "found evidence of delayed reactivation of the grammatical subject, with effects reaching significance only $1000 \mathrm{~ms}$ after the verb", and "delayed or absent reactivation of the filler at the hypothesized gap site." But other studies with healthy and aphasic subjects did not find any "gap-filling effects for passive structures" (Mack et al. 2013). Our analysis is not compatible with this account as Bangla passives do not require syntactic reanalysis. Because the agentive NP occupies the canonical subject position in Bangla passivization.

On the other hand, Head-Driven Phrase Structure Grammar does not recognize that passives involve the movement of NPs, rather passives are distinct from active sentences from the thematic structure. NP-movement based accounts also recognize that passives involve thematic reanalysis i.e. "revision of an initial mapping of thematic roles" (Mack et al. 2013). Eye-tracking studies report when additional cues such as case-marking, or discourse context are provided, subjects interpret sentence-initial NPs as agents which corresponds to the true structure of active (canonical) sentences. Subjects reanalyze the thematic roles upon encountering passive morphology in the sentence i.e. revise the first NP from agent to theme that takes longer reaction times.

Studies also found that complex sentences (such as passives) involve activation in the left inferior frontal gyrus (IFG). Yokoyama et al. (2007) add another perspective to the analysis of the processing of passive sentences in Japanese. They found no greater activation in Broca's area for processing passives, rather greater activation in the left frontal operculum and the inferior parietal lobule were obtained by fMRI studies. This means that impairment of passives might be induced not by lesions to Broca's area, but to the left frontal operculum and/or the inferior parietal lobule (Yokoyama et al. 2007). Japanese passives are morphologically marked by the morpheme rare, while the active verbs remain uninflected. So, the IFG activation may be due to the passive morphology, rather than NP-movement. This leaves yet an unresolved issue whether Japanese and English passives involve similar patterns. Hirotani et al. (2011) also found that passives involved activation in left IFG (pars triangularis) and left posterior superior temporal gyrus (pSTG).

Additionally, passive sentences are usually more difficult to produce. Our experiment also found that passives are the most impaired linguistic items for both aphasic and control groups. It was so, because Bangla passives also require thematic reanalysis compared to active sentences. If we analyze why the production of passives was more impaired by aphasics as well as control subjects, we can find the following general factors affecting the production:

i) The lemmas of passive verbs are assumed to be separate lexical entries in the mental lexicon.

ii) Passive sentences are less frequent in discourse (Dick \& Elman, 2001).

iii) Passive lemmas may have higher activation threshold compared to active verb lemmas (Menn, 1990).

iv) Passive verb lemma retrieval may occur independently of active verb lemma retrieval that may impede the production of passive sentences.

v) The mental grammar does not store an over-learned automatized schema for passive sentences.

vi) Passive sentences usually have more morpho-syntactic elements etc.

In our test, reversibility and non-reversibility did not play any significant role with regard to production impairment of passives. At the first attempt, all the patients tended to produce active sentences, although the written instructions were given explicitly to produce passives. When clarified and told again to produce the sentences with passive markers, though some patients ended up producing passives, many patients produced active sentences over and again or abandoned their attempts altogether after one or two trials. Thus, our findings comply to the fact that passives are non-canonical structures and are more difficult to produce.

\section{CONCLUSION}

The present investigation studied the impairment of non-canonical (passive) structures of Bangla agrammatic speakers, and found that Bangla aphasic agrammatic speakers have severe impairments in non-canonical sentence production. We surveyed ten agrammatic speakers as experimental group as well as the same number of neurologically intact normal Bangla speakers as control group. The control group also produced incorrect responses (though much lesser than the experimental group) while producing non-canonical 
passive sentences. That passives are non-canonical structures and hence involve more difficulty in producing such sentences are substantiated by this study. Both agrammatic and control participants made more errors in Bangla passive sentence production. Not only in Bangla, rather studies on English, German and Japanese agrammatism found agrammatic subjects producing more errors in non-canonical passive sentences. Thus, this cross-linguistic study contributes to the establishing strong theoretical bases concerning aphasiac impairment.

The present study focused on the production of Bangla passive sentences only. But object relative clauses are another significant non-canonical linguistic items cross-linguistically. Comparing the production impairments of Bangla passives and Bangla object relative clauses would yield better analyses. Therefore, further studies on the Bangla object relative clauses would be worth undertaking.

\section{ENDNOTE}

1. We identified one additional type of mistakes for Passives i.e. producing active sentences instead of passives

\section{REFERENCES}

Agnew, Z. K., van de Koot, H., McGettigan, C., \& Scott, S. K. (2014). Do sentences with unaccusative verbs involve syntactic movement? Evidence from neuroimaging. Language, cognition and neuroscience, 29(9), 1035-1045.

Arabatzi, Marina, and Susan Edwards. (2002). Tense and syntactic processes in agrammatic speech. Brain and Language 80.3, 314-327.

Avrutin, S. (2001). Linguistics and agrammatism. Glot international, 5(3), 1-11.

Bastiaanse, R., Hugen, J., Kos, M., \& Van Zonneveld, R. (2002). Lexical, morphological, and syntactic aspects of verb production in agrammatic aphasics. Brain and language 80.2, 142-159.

Bastiaanse, R. (2008). Production of verbs in base position by Dutch agrammatic speakers: Inflection versus finiteness. Journal of Neurolinguistics, 21(2), 104-119.

Bastiaanse, R., Bamyaci, E., Hsu, C. J., Lee, J., Duman, T. Y., \& Thompson, C. K. (2011). Time reference in agrammatic aphasia: A cross-linguistic study. Journal of Neurolinguistics 24.6, 652-673.

Beveridge, Madeleine EL, and Thomas H. Bak. (2011). The languages of aphasia research: Bias and diversity. Aphasiology 25.12, 1451-1468.

Bobaljik, J. D., \& Thráinsson, H. (1998). Two heads aren’t always better than one. Syntax, 1(1), 37-71.

Bornkessel-Schlesewsky, I., \& Schlesewsky, M. (2013). Reconciling time, space and function: a new dorsalventral stream model of sentence comprehension. Brain and language, 125(1), 60-76.

Burchert, F., Meißner, N., \& De Bleser, R. (2008). Production of non-canonical sentences in agrammatic aphasia: Limits in representation or rule application?. Brain and language, 104(2), 170-179.
Chujo, K. (1983). The interrelationships among strategies for sentence comprehension. Japanese Journal of Psychology.

Cho-Reyes, S., \& Thompson, C. K. (2012). Verb and sentence production and comprehension in aphasia: Northwestern Assessment of Verbs and Sentences (NAVS). Aphasiology, 26(10), 1250-1277.

Chomsky, N. (1993). Lectures on government and binding: The Pisa lectures (No. 9). Walter de Gruyter.

Chomsky, N. (1995). The minimalist program. MIT press.

Chujo, K. (1983). The interrelationships among strategies for sentence comprehension. Japanese Journal of Psychology.

Duman, T. Y., \& Bastiaanse, R. (2009). Time reference through verb inflection in Turkish agrammatic aphasia. Brain and Language, 108(1), 30-39.

Engel, S., Shapiro, L. P., \& Love, T. (2018). Proform-antecedent linking in individuals with agrammatic aphasia: A test of the intervener hypothesis. Journal of neurolinguistics, 45, 79-94.

Faroqi-Shah, Y., \& Thompson, C. K. (2004). Semantic, lexical, and phonological influences on the production of verb inflections in agrammatic aphasia. Brain and language, 89(3), 484-498.

Faroqi-Shah, Y., \& Thompson, C. K. (2007). Verb inflections in agrammatic aphasia: Encoding of tense features. Journal of Memory and Language 56.1, 129-151.

Friederici, A. D., Fiebach, C. J., Schlesewsky, M., Bornkessel, I. D., \& Von Cramon, D. Y. (2006). Processing linguistic complexity and grammaticality in the left frontal cortex. Cerebral Cortex, 16(12), 1709-1717.

Friederici, A. D. (2012). The cortical language circuit: from auditory perception to sentence comprehension. Trends in cognitive sciences, 16(5), 262-268.

Friedmann, N., Grodzinsky, Y. (1997). Tense and Agreement in agrammatic production: pruning the syntactic tree. Brain and Language 56, 397-425.

Friedmann, N. (2002). The fragile nature of the left periphery: CP deficits in agrammatic aphasia. In Proceedings of the 18th IATL conference, The Israeli Association for Theoretical Linguistics, The Hebrew University of Jerusalem.

Fyndanis, V., Varlokosta, S., \& Tsapkini, K. (2012). Agrammatic production: Interpretable features and selective impairment in verb inflection. Lingua, 122(10), 1134-1147.

Hartsuiker, R.J., Kolk, H.H.J., Huinck, P. (1999). Agrammatic production of subject-verb agreement: the effect of conceptual number. Brain and Language 69, 119-160.

Hirotani, M., Makuuchi, M., Rüschemeyer, S. A., \& Friederici, A. D. (2011). Who was the agent? The neural correlates of reanalysis processes during sentence comprehension. Human brain mapping, 32(11), 1775-1787.

Hoen, M., Pachot-Clouard, M., Segebarth, C., \& Dominey, P. F. (2006). When Broca experiences the Janus syndrome: An ER-fMRI study comparing sentence comprehension and cognitive sequence processing. Cortex, 42(4), 605-623.

Imtiaz, M. (2013). Sentence Comprehension Problem of Bengali Broca's Aphasics: A Test of the Trace Deletion 
Hypothesis (TDH). [Unpublished Master's Thesis]. University of Dhaka, Dhaka, Bangladesh.

Kemmerer, D. (2012). The Cross-Linguistic Prevalence of SOV and SVO Word Orders Reflects the Sequential and Hierarchical Representation of Action in Broca's Area." Language and Linguistics Compass 6.1, 50-66.

Kinno, R., Kii, Y., Kurokawa, S., Owan, Y., Kasai, H., \& Ono, K. (2017). Effects of word order and morphological information on Japanese sentence comprehension in nonfluent/agrammatic variant of primary progressive aphasia. Journal of Neurolinguistics 44, 107-119.

Kok, P., van Doorn, A., \& Kolk, H. (2007). Inflection and computational load in agrammatic speech. Brain and Language, 102(3), 273-283.

Lee, J., \& Thompson, C. K. (2005). Functional categories in agrammatic speech. LSO working papers in linguistics, $5,107$.

Levelt, W. J. M, C. Brown, \& Hagoort, P. (1999). The neurocognition of language. Language Production: A blueprint of the speaker (ed.) C. Brown and P. Hagoort

Mack, J. E., Meltzer-Asscher, A., Barbieri, E., \& Thompson, C. K. (2013). Neural correlates of processing passive sentences. Brain sciences, 3(3), 1198-1214.

Makuuchi, M., Grodzinsky, Y., Amunts, K., Santi, A., \& Friederici, A. D. (2013). Processing noncanonical sentences in Broca's region: reflections of movement distance and type. Cerebral Cortex, 23(3), 694-702.

Mehri, A., Ghorbani, A., Darzi, A., Jalaie, S., \& Ashayeri, H. (2016). Comparing the production of complex sentences in Persian patients with post-stroke aphasia and non-damaged people with normal speaking. Iranian journal of neurology, 15(1), 28.

Menn, L. (2009). Child language, aphasia, and general psycholinguistics. na.

Nakayama, M. (1995). Scrambling and probe recognition. Japanese sentence processing, ed. by R. Mazuka, and N. Nagai, 257-273.

Nanousi, V., Masterson, J., Druks, J., \& Atkinson, M. (2006). Interpretable vs. uninterpretable features: Evidence from six Greek-speaking agrammatic patients. Journal of Neurolinguistics, 19(3), 209-238.

Newman, S. D., Ikuta, T., \& Burns Jr, T. (2010). The effect of semantic relatedness on syntactic analysis: an fMRI study. Brain and language, 113(2), 51-58.

Ouhalla, J. (1993). Functional categories, agrammatism and language acquisition. Linguistische Berichte, (143), $3-36$.
Price, C. J., \& Crinion, J. (2005). The latest on functional imaging studies of aphasic stroke. Current opinion in neurology, 18(4), 429-434.

Rispens, J., Bastiaanse, R., \& Van Zonneveld, R. (2001). Negation in agrammatism: a cross-linguistic comparison. Journal of Neurolinguistics, 14(1), 59-83.

Roland, D., Dick, F., \& Elman, J. L. (2007). Frequency of basic English grammatical structures: A corpus analysis. Journal of memory and language, 57(3), 348-379.

Röder, B., Stock, O., Neville, H., Bien, S., \& Rösler, F. (2002). Brain activation modulated by the comprehension of normal and pseudo-word sentences of different processing demands: a functional magnetic resonance imaging study. Neuroimage 15.4, 1003-1014.

Santi, A., \& Grodzinsky, Y. (2010). fMRI adaptation dissociates syntactic complexity dimensions. Neuroimage, 51(4), 1285-1293.

Schwartz, M. F., Linebarger, M. C., Saffran, E. M., \& Pate, D. S. (1987). Syntactic transparency and sentence interpretation in aphasia. Language and Cognitive Processes, 2(2), 85-113.

Shapiro, T., Sherman, M., Calamari, G., \& Koch, D. (1987). Attachment in autism and other developmental disorders. Journal of the American Academy of Child \& Adolescent Psychiatry 26.4, 480-484.

Tamanna, S. P. (2017). Production and comprehension deficit in Broca's Aphasia: A test in Bengali (Master's thesis, The University of Bergen).

Tamaoka, K., Sakai, H., Kawahara, J. I., \& Miyaoka, Y. (2003). The effects of phrase-length order and scrambling in the processing of visually presented Japanese sentences. Journal of Psycholinguistic Research 32.4, 431-454.

Varlokosta, S., Valeonti, N., Kakavoulia, M., Lazaridou, M., Economou, A., \& Protopapas, A. (2006). The breakdown of functional categories in Greek aphasia: Evidence from agreement, tense, and aspect. Aphasiology 20.8, 723-743.

Vigneau, M., Beaucousin, V., Herve, P. Y., Duffau, H., Crivello, F., Houde, O.,... \& Tzourio-Mazoyer, N. (2006). Meta-analyzing left hemisphere language areas: phonology, semantics, and sentence processing. Neuroimage 30.4,1414-1432.

Wenzlaff, M., \& Clahsen, H. (2004). Tense and agreement in German agrammatism. Brain and language, 89(1), 57-68.

Yokoyama, S., Watanabe, J., Iwata, K., Ikuta, N., Haji, T., Usui, N.,... \& Horie, K. (2007). Is Broca's area involved in the processing of passive sentences? An event-related fMRI study. Neuropsychologia, 45(5), 989-996. 